\title{
EFFECTS OF REFIRING PROCESSES ON ELECTRICAL AND STRUCTURAL PROPERTIES OF THICK-FILM RESISTORS
}

\author{
A. CATTANEO \\ M. MARELLI D.S.E. Via F. Filzi 1, 27100 Pavia (Italy) \\ and \\ M. PRUDENZIATI \\ Istituto di Fisica, Via Campi, 41100 Modena (Italy)
}

(Received A pril 27, 1979)

\begin{abstract}
In order to analyze the influence of the refiring processes in thick film resistors, electrical and structural properties of resistors, refired up to ten times, through the original profile, were compared.

The apparent sheet resistivity and TCR were measured as a function of the number of the firing cycles, and these electrical characteristics analyzed in connection with observations of structural and chemical modifications evidenced through X-ray diffraction, SEM inspection and microprobe analysis.

Marked effects were observed, such as segregation of the conductive grains in the resistor thickness, chemical reactions of glass-modifiers with glass, crystallization of new phases, together with substrate/thick-film interactions.

It is found that in some cases (e.g. for DP 1400 resistor series) the phenomena induced by refiring are few and simple enough for us to interprete the corresponding electrical modifications, while in other systems the presence of many competitive chemicophysical effects inhibit their unambiguous correlation with the electrical properties of these resistors.
\end{abstract}

\section{INTRODUCTION}

Close control of the electrical properties of thick-film resistors is of primary importance in development of passive networks for hybrid microelectronics. It is well known that ohmic values and temperature coefficients of resistance (TCR) are correlated to the firing parameters (time and temperature) used in ink-processing ${ }^{1-3}$ so that close control of the firing cycle is essential to give reliable thick-film resistors.

Data of sheet resistivity and TCR changes due to variations in the firing thermal cycle of resistors have been frequently reported ${ }^{2-5}$ but poor information is available on phenomena responsible for these electrical-properties changes.

In order to complete a previous paper where we pointed out the influence of metal-migration from screen-and-fired terminations on the electrical properties of thick-film-resistors, ${ }^{6}$ the present paper concerns the analysis of effects due to refiring processes unaffected by terminations phenomena.

For this study we shall use two different commercial ink-systems, a ruthenate-based ink-family (DP 1400) and a ruthenium-oxide/ink-family (Plessey
SRD). However the study was not intended as a product evaluation and comparison but as an exemplification of effects and methods of detection.

Within each series we analyzed the behaviour of resistors with nominal sheet resistivities in the range $10^{2}$ to $10^{6} \mathrm{ohm} / \square$ but in the present work we discuss only a few results, reporting the most representative data in order to understand better the possible events occurring in refiring processes.

It will be shown that resistors of the same series (e.g. Plessey SRD) but with different nominal resistivities are differently affected by refiring processes. This is a consequence of the fact that inks of the same family, with the same series number, do not really represent a unique physical system where the conductor/frit ratio is the most important parameter, but each ink is characterized by glass-modifier in order to get a better control of the desired electrical properties of resistors.

Moreover it will be shown that in some cases (e.g. DP 1400) the physico-chemical phenomena induced by refiring processes are few and simple enough to be unambiguously analyzed and correlated to electrical properties changes. On the other hand, 
in other cases, (e.g. Plessey SRD) several competitive effects are detected so that a simple performance of their influence on the electrical properties of resistors is not possible.

\section{SAMPLES PREPARATION AND EXPERIMENTAL METHODS}

Resistors were prepared according to the usual procedures of screening, drying and firing well known in microelectronic hybrid circuits. ${ }^{1}$ Both alumina and beryllia substrates were used and in some cases, in order to increase electrical and structural effects induced by refiring, resistors were refired several (up to ten) times without any modification of the recommended firing cycle. Samples were fired either upward and downwards but in many cases, no difference was observed.

A four-point probe technique ${ }^{7}$ was used for the measurement of the sheet resistivity, while for the measurements of resistance vs temperature, resistors were provided with silver epoxy-resins contacts, cured at room temperature.

After the preparation of the resistors, electrical measurements were performed. Sheet-resistivity data reported are mean values obtained on at least 30 samples, with an equal number of firing cycles. The composition and structure analysis of the thick-film materials included a semiquantitative chemical analysis with energy-dispersive electron microprobe, SEM inspection and X-ray diffractometry.

\section{RESULTS AND DISCUSSION}

\subsection{Du Pont 1400 Series}

Figure 1 shows the sheet-resistivity dependence in the refiring-cycles in some resistors of this series. The ratio $\left(R_{n}-R_{1}\right) / R_{1}$ is plotted as a function of the number of firing cycles where $R_{n}$ is the resistivity of $n$-times refired samples and $R_{1}$ the resistivity of normal fired samples. It is evident that sheet resistivity is strongly affected by heat treatment, particularly in the first stages of refiring and at higher nominal resistivities. It should be noted that data are independent of the nature of the substrate (alumina or beryllia) and that resistivity tends to reach a stable value after many firing cycles.

Changes in TCR are also induced by refiring processes. Figure 2 shows the temperature dependence of resistance in $10 \mathrm{kohm} / \square$ resistors after one and ten firing cycles. Resistance is normalized to $R_{\mathrm{min}}$, the

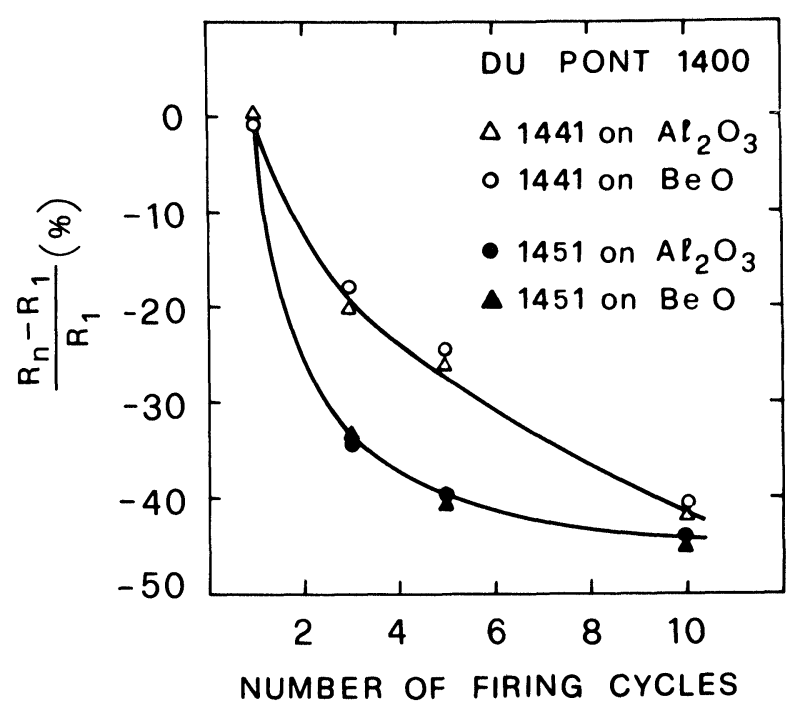

FIGURE 1 Effects of refiring processes on sheet resistivity of DP 1441 and 1451 resistors on alumina and beryllia substrates.

value corresponding to the temperature $T_{\min }$ where TCR vanishes, according to a well known characteristic $^{8-11}$ of thick-film resistors. By increasing the firing cycles, $T_{\min }$ shifts at lower temperatures. Structure and composition analyses account for these observations of electrical behaviour.

Figure 3a shows the X-ray diffraction pattern of 1441 resistors after one firing cycle. Typical lines of the pyroclore structure are evident, pertaining either to $\mathrm{Bi}_{2} \mathrm{Ru}_{2} \mathrm{O}_{7}$ or $\mathrm{Pb}_{2} \mathrm{Ru}_{2} \mathrm{O}_{7-\mathrm{x}}$ (with $\mathrm{O}<x<1$ ). Cell parameters of these compounds are so similar, that a clear identification with this technique ${ }^{12,15}$ is very difficult. On the other hand microprobe analysis gives a high Bi-concentration in these resistors and

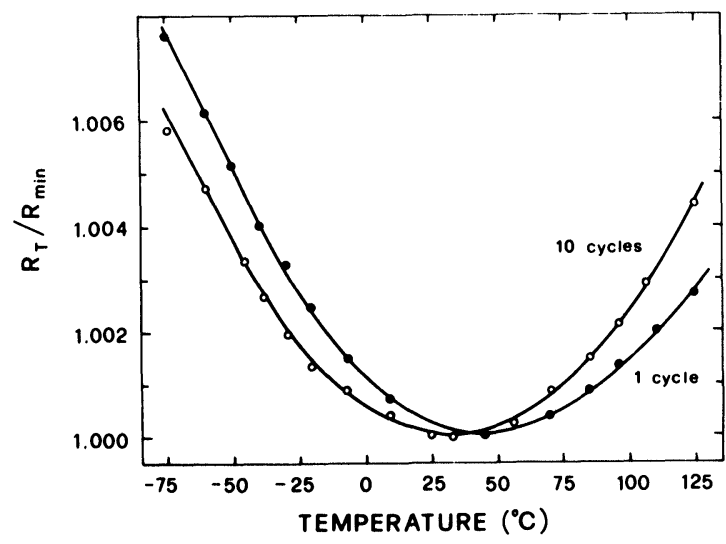

FIGURE 2 Temperature-dependence of resistance in DP 1441 resistors on alumina after 1 and 10 firing cycles. 

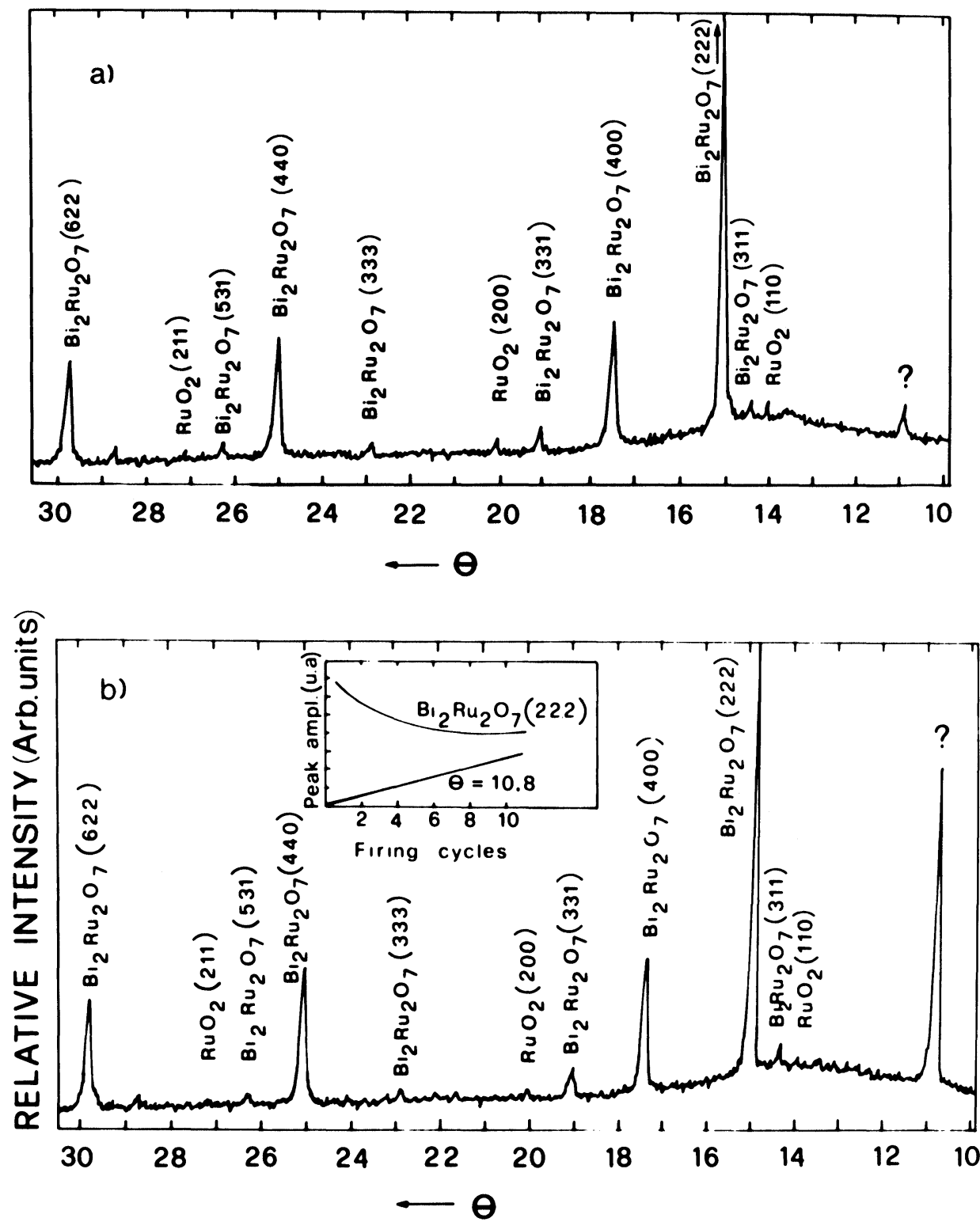

FIGURE 3 X-ray diffraction patterns of DP 1441 resistors (a) after one and (b) after 10 firing cycles. In the insert the relative intensity of some diffractometric lines as a function of the number of firing cycles is reported.

the presence of $\mathrm{Bi}_{2} \mathrm{Ru}_{2} \mathrm{O}_{7}$ is likely. Moreover diffraction patterns show the presence of $\mathrm{RuO}_{2}$ grains, probably coming from the decomposition of ruthenate during the firing cycles, and where it is also present the dissolution of bismuth-oxide in glass.

Finally a phase not identified give a peak at $\theta=10.8^{\circ}$.

After ten firing cycles, the diffraction pattern of the same resistor appears quite modified. The most apparent effect is the increase in height of the peak at $\theta=10.8^{\circ}$. This peak is systematically present in resistors of this series screen-and-fired on alumina substrates and its relative intensity linearly increases with the number of firing cycles (see insert of Figure 3) but it does not appear if the same resistors are fired on beryllia substrates. This observation suggests an interaction of resistors with $\mathrm{Al}_{2} \mathrm{O}_{3}$ substrates, with a subsequent crystallization of a new phase, probably a silicate compound, ${ }^{12}$ which 
can not be easily identified due to the lack of other significant diffraction peaks. On the other hand the identification of this phase is not important for the interpretation of the previously described electrical properties since these parameters are independent of the phase itself and are the same in resistors printed on different substrates.

Comparison of Figures 3(a) and (b) also shows a change in the relative heights of peaks due to ruthenate-phase. This effect appears in resistors screen-and-fired either on alumina and beryllia, while the dependence of the (222) ruthenate line on the number of firing cycles is analogous to that of resistivity as observed by comparing Figure 1 with the insert of Figure 3 . These findings suggest a close correlation of electrical phenomena with structural phenomena induced by refiring of these resistors.

Moreover we found that grain sizes of $\mathrm{Bi}_{2} \mathrm{Ru}_{2} \mathrm{O}_{7}$ and $\mathrm{RuO}_{2}$, inferred by the diffraction-line broadening, ${ }^{12}$ have no apparent change after refiring, so phenomena of sinterization or dissolution of conductive grains ${ }^{13}$ can be rejected.

It seems to be unlikely that changes in relative intensity of ruthenate-peaks could be ascribed to substitution of lead by bismuth in the glass. ${ }^{14,15}$

A simpler interpretation, supported by SEM and microprobe analysis, is as follows. During the refiring process, film components rearrange so that upper layers in the film become richer in glass and less rich in conductive grains than before the refiring processes.

In this case, the decrease of ruthenate peak heights is a mere consequence of the reduction of ruthenate in the upper layers of the film, investigated by the diffracted X-ray beam.

This phenomenon actually emerges from a thickness not greater than few microns as a consequence of the high density and high mean atomic number of the film, inferred by the microprobe-analysis data. Interesting information comes from microprobe and SEM analyses. The main components of the resistors of DP 1400 series are, in order of relative concentration, lead, silicon, bismuth, ruthenium, aluminium, cadmium and traces of additives e.g. platinum in 1421, gallium in 1441. Bismuth is absent in 1461 where the main conductive component is $\mathrm{Pb}_{2} \mathrm{RuO}_{6}$.

In refired resistors the surface concentration (detected with microprobe) of silicon is higher and of ruthenium is lower than in once-fired samples. Moreover in resistors on alumina substrates an increased aluminium concentration in refired samples is also detected which can be ascribed to the silicate growth and the substrate interaction suggested by X-ray diffractometry.

These results support the conductive rearrangement in the film, also evidenced by SEM inspection of the resistor slightly etched in HF in order to remove a thin layer of glass (Figures $4 a$ and $b$ ). In fact the surface of ten-times refired resistors appears less compact and dense than that of a once-fired sample.

The rearrangement of the film component, with segregation of conductive grains toward the substrate accounts for the electrical-characteristic changes induced in these resistors by refiring.

Let us consider first the sheet-resistivity. This is given by a parallel combination of the resistivities of different layers in the depth of the film. On the other hand, it is well known ${ }^{4,13,14,16}$ that resistivity in thick-film resistors is a superlinear function of the inverse of conductive concentration in the film.

When conductive grains thicken in the film, there is a strong increase of resistivity in the upper layers and, vice-versa, a strong decrease of resistivity in the lower layers. The mean value of the resistivity of all

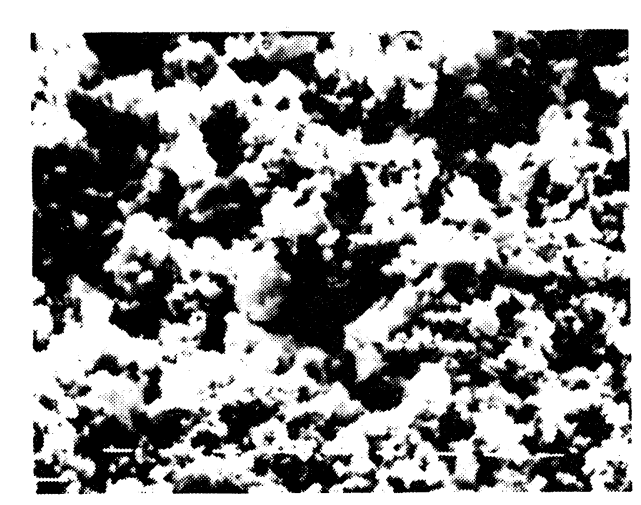

(a)

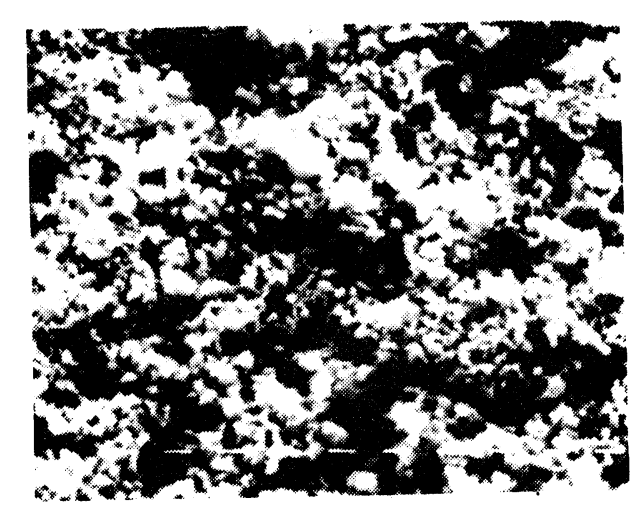

(b)

FIGURE 4 SEM pictures of DP 1441 resistors after slight etching in HF. (a) one firing cycle; (b) ten firing cycles. 
the layers has to remain fixed to the initial value of the whole film. Consequently the sheet resistivity of refired resistors decreases, since the resistance of a parallel-resistor network, whose mean value is fixed, has a maximum value when all resistors have equal resistances.

Let us consider now the temperature-dependence of resistance. The rearrangement of the conductive component accounts for data in Figure 2. In fact it has been shown ${ }^{11}$ that in a "blended system" there exists a direct relationship between the conductorconcentration and the temperature $T_{\min }$ at which TCR vanishes. The higher is the concentration, the lower is the $T_{\min }$ value as expected from the tunnelling conduction model for thick-film resistors. ${ }^{10}$

Data in Figure 2 are then consistent with an increase of conductor concentration taking into account that the measurement evidences the behaviour of lower-resistivity layers in the film.

\subsection{Plessey SRD Series}

Figure 5 shows the sheet resistivity changes induced by refiring of resistors of this series. Substantial differences in the behaviour of $10 \mathrm{kohm} / \square$ and $100 \mathrm{kohm} / \square$ show the singularity in the behaviour of different inks of the same nominal series. Figure 6 shows the temperature-dependence of resistance in a $10 \mathrm{kohm} / \square$ resistor fired once and ten-times

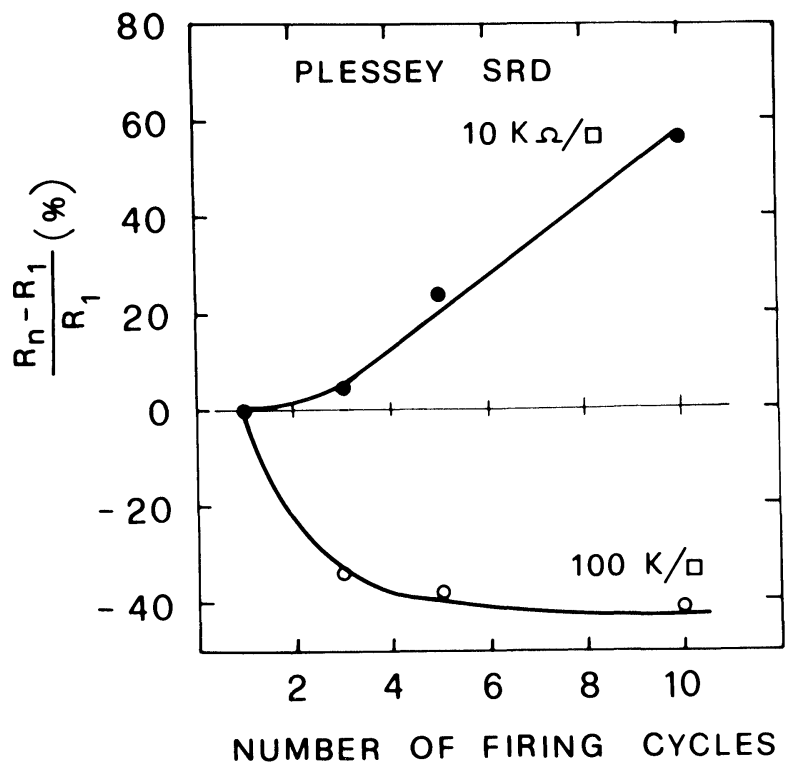

FIGURE 5 Effect of refiring on the sheet resistivity of SRD Plessey resistors.

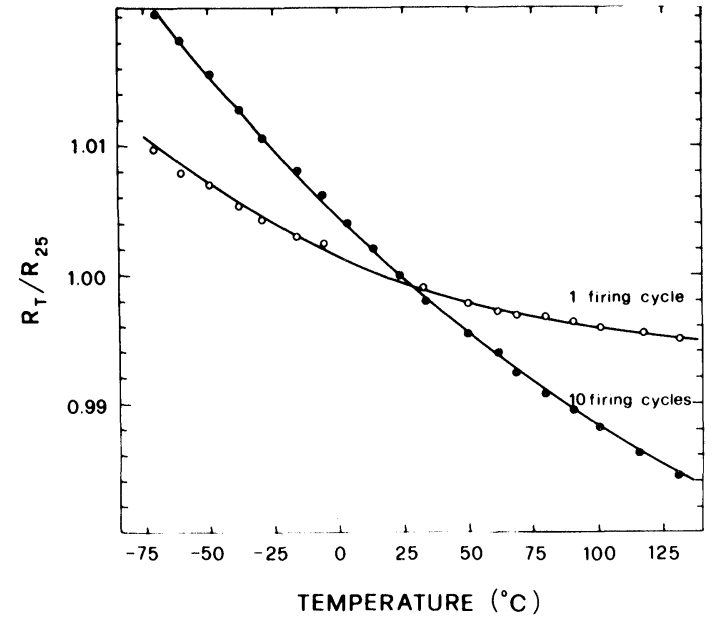

FIGURE 6 Temperature-dependence of resistance in SRD Plessey resistors $10 \mathrm{kohm} / \square$ after 1 and 10 firing cycles.

respectively. In these cases the TCR does not vanish at a temperature in the considered range, so that the resistance values $R_{\mathrm{T}}$ are normalized to the resistance measured at $25^{\circ} \mathrm{C}$. The increase of TCR induced by refiring processes is evident. This phenomena, together with the increased sheet resistivity, is consistent with previous experimental findings. ${ }^{11}$

Figure 7 shows the X-ray diffraction patterns of $10 \mathrm{kohm} / \square$ resistors of this series. The cubic $\mathrm{RuO}_{2}$ and $\alpha-\mathrm{Al}_{2} \mathrm{O}_{3}$ structures can easily be recognized and an orthorombic phase is evident which microprobe analysis identified as $\mathrm{PbTiO}_{3}$. After ten firing cycles, the same resistors give modified X-ray patterns, essentially since peaks due to the $\mathrm{PbTiO}_{3}$ phase have greater heights. On the other hand, no change is evident in the $\mathrm{RuO}_{2}$ peaks. Analogous features were obtained from $100 \mathrm{kohm} / \square$ resistors in this series. In this case the $\mathrm{PbTiO}_{3}$ phase, absent in once-fired resistors, appears only after 3 firing cycles together with $\alpha-\mathrm{Al}_{2} \mathrm{O}_{3}$.

The main components of these resistors in order of relative concentration are lead, ruthenium, silicon, aluminium, titanium, potassium. In $100 \mathrm{kohm} / \square$ resistors, traces of gallium are also detected.

Element concentration changes in resistors of different resistivity, the $\mathrm{Ru} / \mathrm{Pb}$ ratio as well as the $\mathrm{Si} / \mathrm{Ti}$ ratio is higher in the $10 \mathrm{kohm} / \square$ resistors than in the $100 \mathrm{kohm} / \square$ resistors whilst the ratios $\mathrm{Ru} / \mathrm{Al}$ and $\mathrm{Ru} / \mathrm{K}$ are lower in $10 \mathrm{kohm} / \square$ resistors than in $100 \mathrm{kohm} / \square$ resistors. Microprobe and SEM electron beams incident on these resistors cause the appearance of areas with secondary electron emission of intensity and density which increases by increasing the exposure time. 

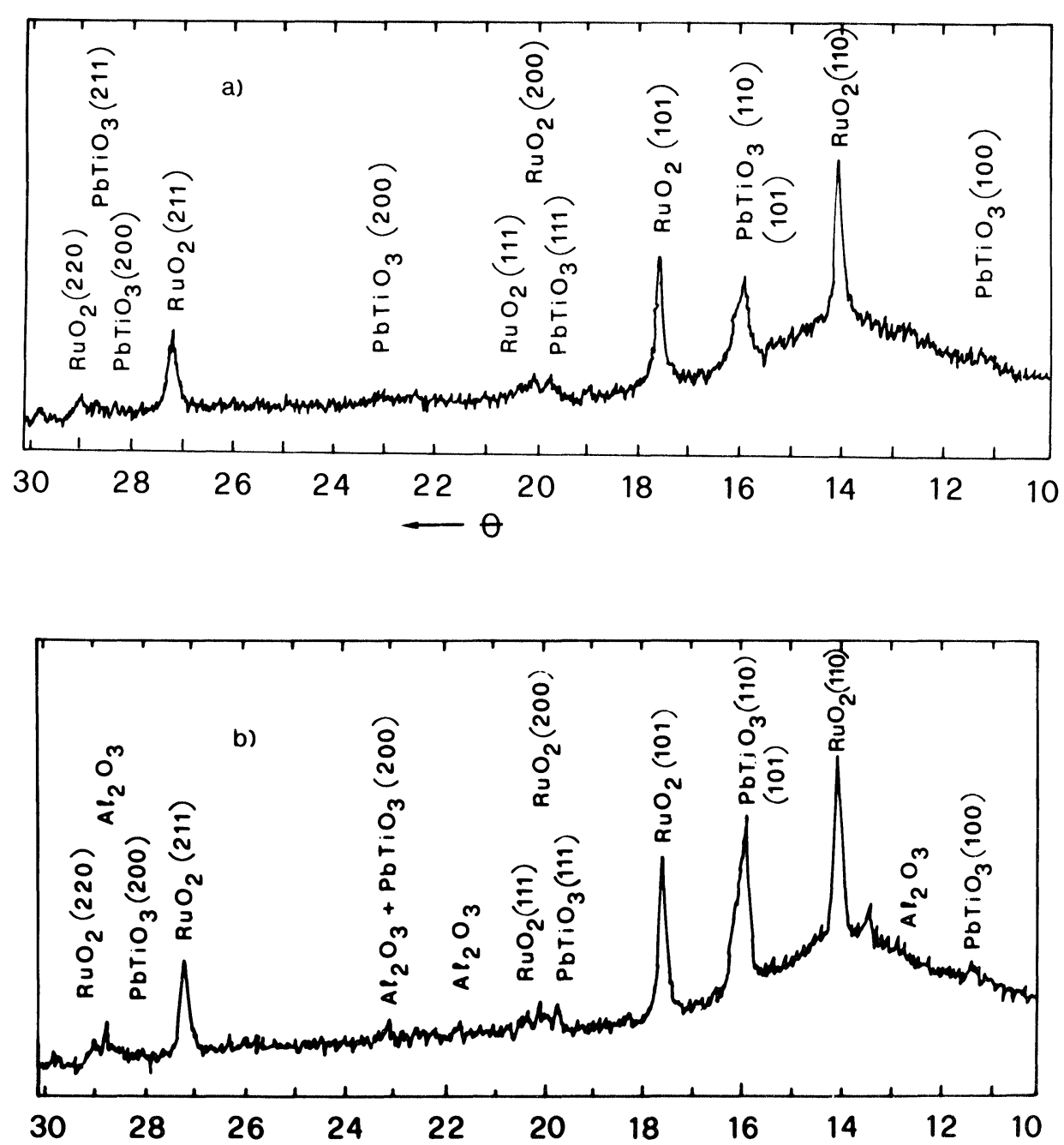

FIGURE 7 X-ray diffraction pattern of SRD Plessey resistors $10 \mathrm{kohm} / \square$ (a) 1 firing cycle and (b) 10 fïing cycles.

After some minutes the picture shown in Figures 8 are obtained. Microprobe analysis indicates that these areas correspond to potassium migration. Different distributions of these areas are observed in once fired and refired resistors (Figures $8 a$ and $b$ ). This effect is more noticeable in $100 \mathrm{kohm} / \square$ than in $10 \mathrm{kohm} / \square$ resistors of this series.

Summing up the experimental findings we note that refiring processes cause several changes in the structure and composition of this series: titanium and aluminium coming from the glass matrix induce crystallization of lead titanate and corundum, potassium migrate in the film.

These effects are common to $10 \mathrm{kohm} / \square$ and $100 \mathrm{kohm} / \square$ resistors, but are different in intensity, so that they have to be competitive in order to give such differing electrical changes as observed.

It is known that glass modifiers strongly affect the resistivity and TCR of thick-film resistors. ${ }^{17}$ Hence it is not surprising that modifications of the glass matrix are followed by noticeable changes in electrical properties of these resistors.

Unfortunately our knowledge of the role played by glass modifiers in thick-film resistor conduction mechanisms is not complete.

An attempt to correlate electrical and structural effects in this specific situation is at the present moment very difficult. All we can suggest is that more effects tend to change the resistance and TCR but their identification could be unreliable. 


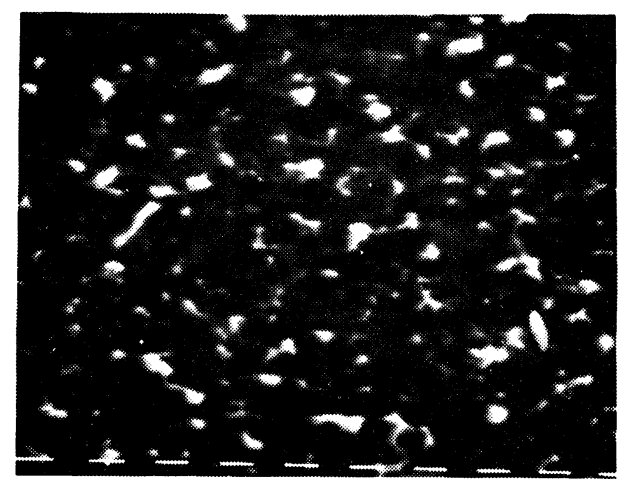

(a)

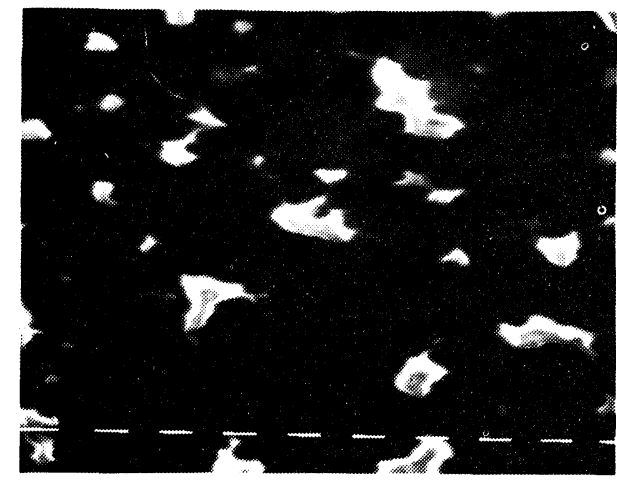

(b)

FIGURE 8 SEM pictures of $100 \mathrm{kohm} / \square$ Plessey SRD resistors after (a) 1 and (b) 10 firing cycles.

\section{CONCLUSIONS}

We illustrated some examples of refiring effects which can happen in thick-film resistors unaffected by the presence of thick-film conductor terminations. These examples focalize the differences of behaviour in different systems and in resistors obtained by inks of the same series. Moreover we identified some of the parameters to be controlled in the description of thick-film resistors. The research has shown that refiring processes give macroscopic variations of structure and composition of the films easily detected by techniques such as X-ray diffractometry, electron microprobe analysis and scanning electron microscopy. Moreover the cases here reported suggest that the stability of some thick-film resistor systems is strongly affected by the glass-matrix modification induced by thermal treatment and show the need of further research to obtain information on the rôle of glass modifier on the electrical-properties of thickfilm resistors.

\section{REFERENCES}

1. Handbook of Thick Film Hybrid Microcircuits Ed. C. A. Harper, McGraw Hill, (New York, 1974).

2. Birox 1400 Series Resistors, Du Pont Data Serial A-83691-10/72.

3. L. C. Hoffman and M. J. Popwich, Solid State Technol. 33 (Jan. 1971).

4. H. C. Angus and P. E. Gainsbury, Electron. Components. 9, 84 (1968).

5. Y. Takeda and M. Haradome, IEEE Trans. PHP-9, 115 (1973).

6. A. Cattaneo, M. Cocito, F. Forlani and M. Prudenziati, ElectroComp. Sci. Technol. 4, 205 (1977).

7. L. B. Valdes, Proc. IRE 42, 420 (1954).

8. E. H. Melan, Microelectron Reliab. 6, 53 (1967).

9. A. Kusy, Thin Solid Films 17, 345 (1973).

10. F. Forlani and M. Prudenziati, Electro Comp. Sci. Technol. 3, 77 (1976).

11. M. Prudenziati, Alta Frequenza, 46, 287 (1977).

12. A. Taylor "X-ray Metallography" John Wiley and Sons, (New York, 1961) and ASTM Powder Diffraction File.

13. R. W. Vest. Final Technic. Report, Purdue University (1975).

14. G. E. Pike and C. H. Seager, SAND 76-0558 Report (1977)

15. J. S. Shah and W. C. Hahan, Electr. Comp. Conf., Aneheim-USA, (1978).

16. P. R. Van Loan, Ceram. Bull. 51, 231 (1972) e M. V. Coleman Radio and Electron. Eng. 45, 121 (1975).

17. S. Fu, J. Liang, T. Shiramatsu and T. Wu. Mater. Res Bull. 12, 569 (1977). 

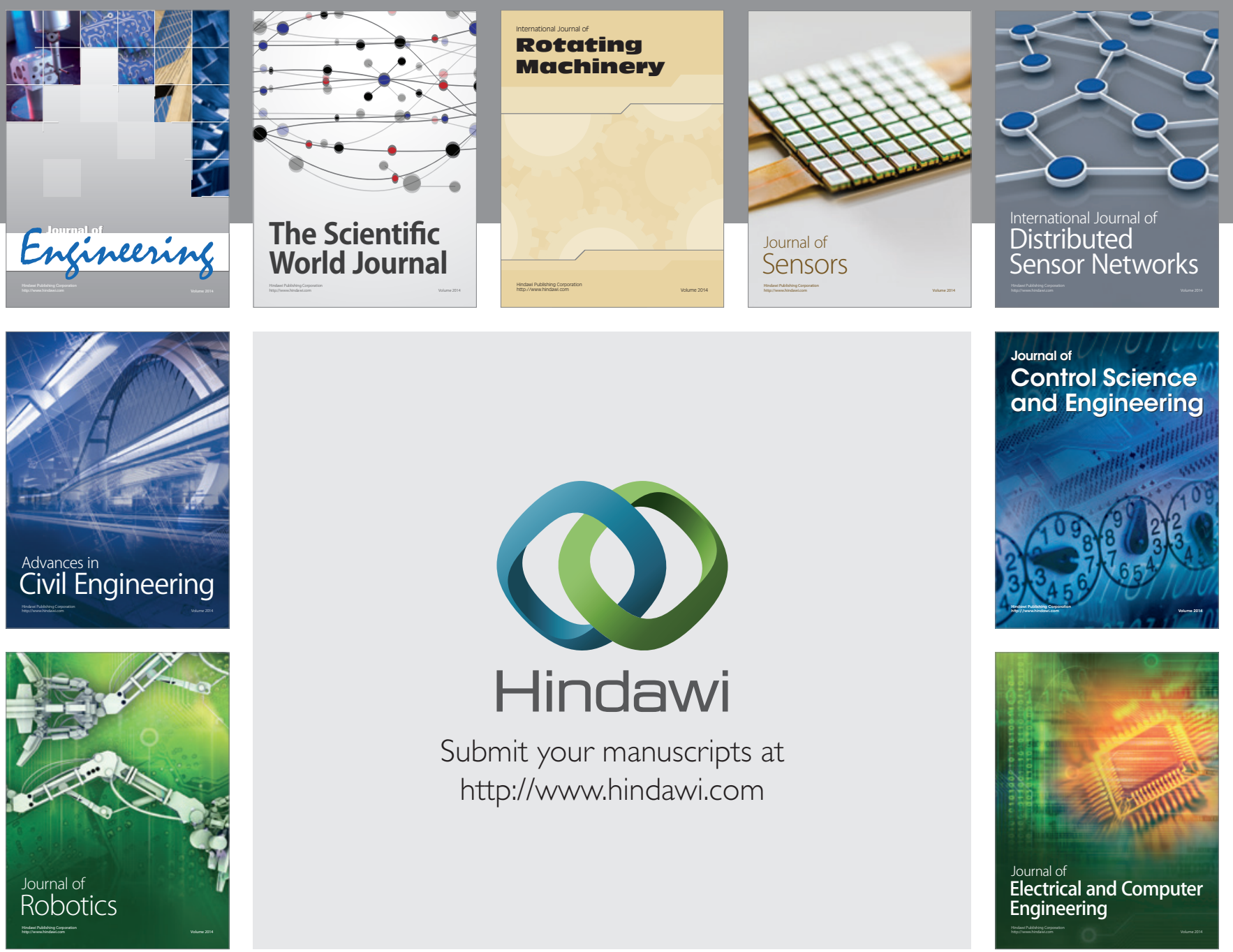

Submit your manuscripts at

http://www.hindawi.com
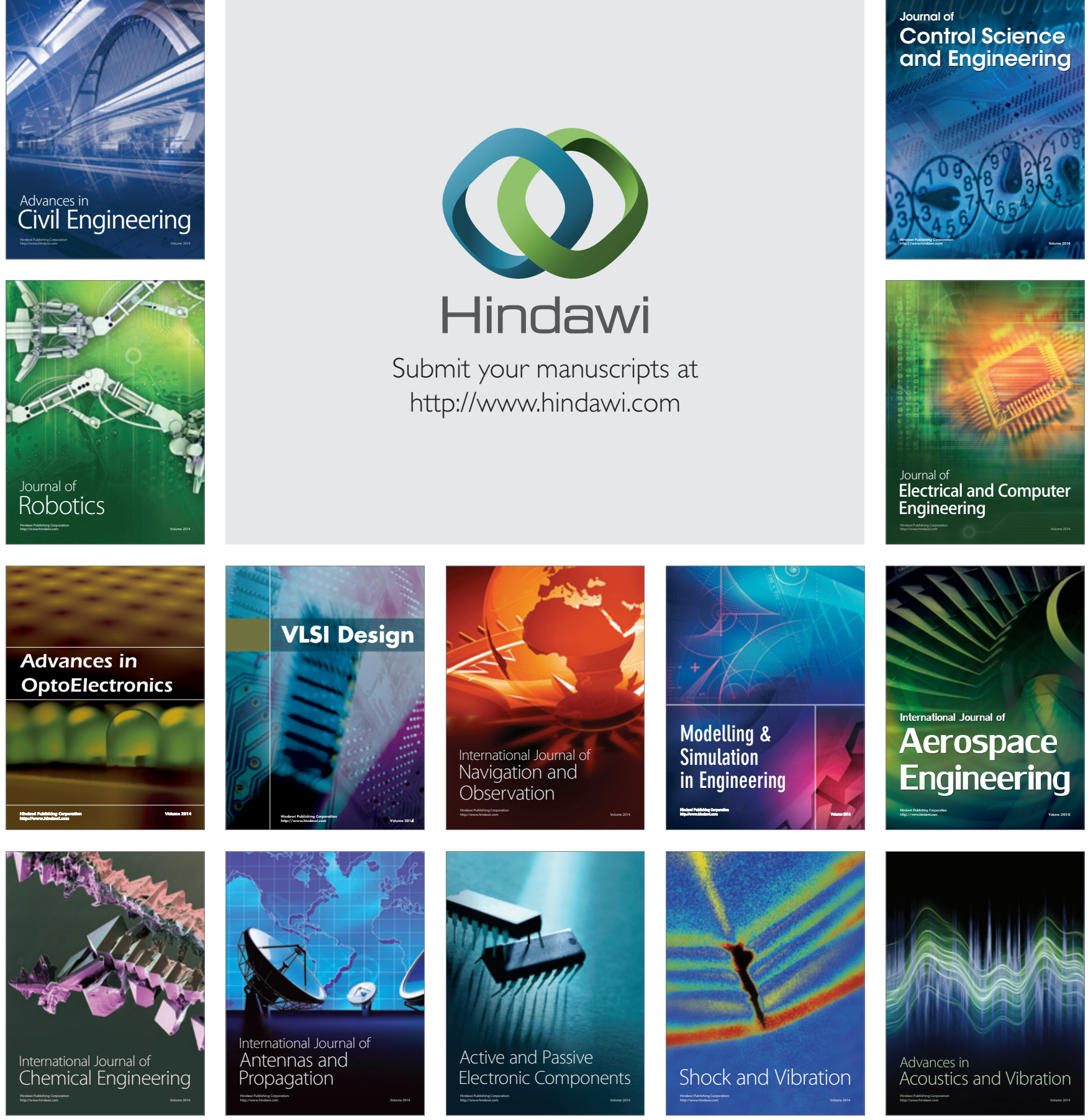